\title{
Acute Psychosis Precipitated by Urinary Tract Infection in a Patient with Gliosis of the Basal Ganglia
}

\author{
Philip Lee, Filip Oleszak, Aditya Nihalani, Vinodkumar Velayudhan, Isabel M. McFarlane* \\ Department of Internal Medicine, State University of New York, Downstate Medical Center, Brooklyn, NY 11203 USA. \\ *Corresponding author: Isabel.McFarlane@downstate.edu
}

Received August 19, 2019; Revised September 26, 2019; Accepted October 08, 2019

\begin{abstract}
Background: Urinary tract infections (UTI) have been found to be associated with a variety of neuropsychiatric disorders, and could play a role in the pathophysiology of relapse of affective and nonaffective psychosis. In addition, prior history of infarction in areas of the brain such as the cerebellum, basal ganglia, and mid-brain have been reported in patients with new onset psychotic symptoms. Case presentation: A 29-year-old woman was brought to the hospital with acute mental status changes and signs of sepsis. Infectious work-up was initiated including blood cultures, brain imaging, lumbar tap and urinalysis. Brain MRI revealed abnormalities in the basal ganglia and the urinalysis revealed signs of a urinary tract infection (UTI). Further history revealed episodes of mania and depression compatible with bipolar disorder with psychotic features that had acutely worsened. The patient's condition improved with intravenous antibiotics and the introduction of anti-psychotics. She was discharged in stable condition with outpatient psychiatric follow-up. Conclusion: Infectious diseases (UTIs in particular) are not only more prevalent among patients with acute relapse of psychiatric disorders, but have also been found to have triggered acute psychosis among stable psychiatric patients. Organic brain lesions must be thoroughly investigated among patients presenting with new psychiatric disorders in order to initiate appropriate therapy to control the symptoms.
\end{abstract}

Keywords: acute psychosis, urinary tract infection, mania, schizophrenia, affective psychosis, bipolar disorder with psychotic features, gliosis, microbiome, macrophages, blood brain barrier, inflammation, delusion, hallucination, mood disorder

Cite This Article: Philip Lee, Filip Oleszak, Aditya Nihalani, Vinodkumar Velayudhan, and Isabel M. McFarlane, "Acute Psychosis Precipitated by Urinary Tract Infection in a Patient with Gliosis of the Basal Ganglia.” American Journal of Medical Case Reports, vol. 7, no. 12 (2019): 329-333. doi: 10.12691/ajmcr-7-12-7.

\section{Background}

Infections have been recognized as triggers of acute episodes of psychosis among patients with neuropsychiatric disorders [1]. On the other hand, schizophrenia patients who develop acute infections bear increased morbidity and mortality [2]. In particular, UTI are found at a high prevalence rate among patients with a variety of psychiatric presentations [2]. Most recently, among patients with psychiatric disorders, UTI occurring during pregnancy was found to confer a higher risk nonaffective psychosis to the offspring [3].

It has become apparent that alterations in the microbiome makeup, due to the presence of a precisely timed pathogen exposure in a person with genetically encoded altered immunity, may lead to an imbalance that have damaging consequences for the central nervous system (CNS) [4]. Furthermore, brain imaging abnormalities such as cortical atrophy, encephalomalacia, hypodensities suggesting prior infarction and gliosis have been reported in patients with psychosis. Brain lesions have been postulated to alter the normal pathways leading to functional disturbances in the cortex regions that affect normal perception of reality affecting sensory, visual and behavior [5].

\section{Case Presentation}

A 29-year-old highly educated woman with no previously known medical or psychiatric history presented to the hospital after acute onset of altered mental status. The patient appeared disheveled, incoherent and preoccupied about religious beliefs. She exhibited pressure speech and was responding to internal stimuli. There was no headache, photophobia, nausea, vomiting, sick contacts, exposure to illegal drugs or toxins, recent viral illness or preceding episodes of similar presentation. On examination, she was found to be febrile (100.7 F), tachycardic (148 BPM), hypertensive $(145 / 89 \mathrm{mmHg})$, with a respiratory rate of 18 . Her BMI was $>40 \mathrm{Kg} / \mathrm{m}^{2}$. The physical exam was unremarkable. There was no meningeal signs, cranial nerves were intact except for horizontal nystagmus.

Blood cultures were obtained. Laboratory data revealed leukocytosis $\left(17.65\right.$ cells $\left./ \mathrm{mm}^{3}\right)$ with neutrophilic predominance with $86 \%$ neutrophils, $10.2 \%$ lymphocytes, $2.8 \%$ monocytes, $0.3 \%$ eosinophils, $0.3 \%$ basophils. In 
addition, patient had a hemoglobin of $11.1 \mathrm{~g} / \mathrm{dL}$ and platelets of $452 \mathrm{~K} / \mathrm{uL}$.

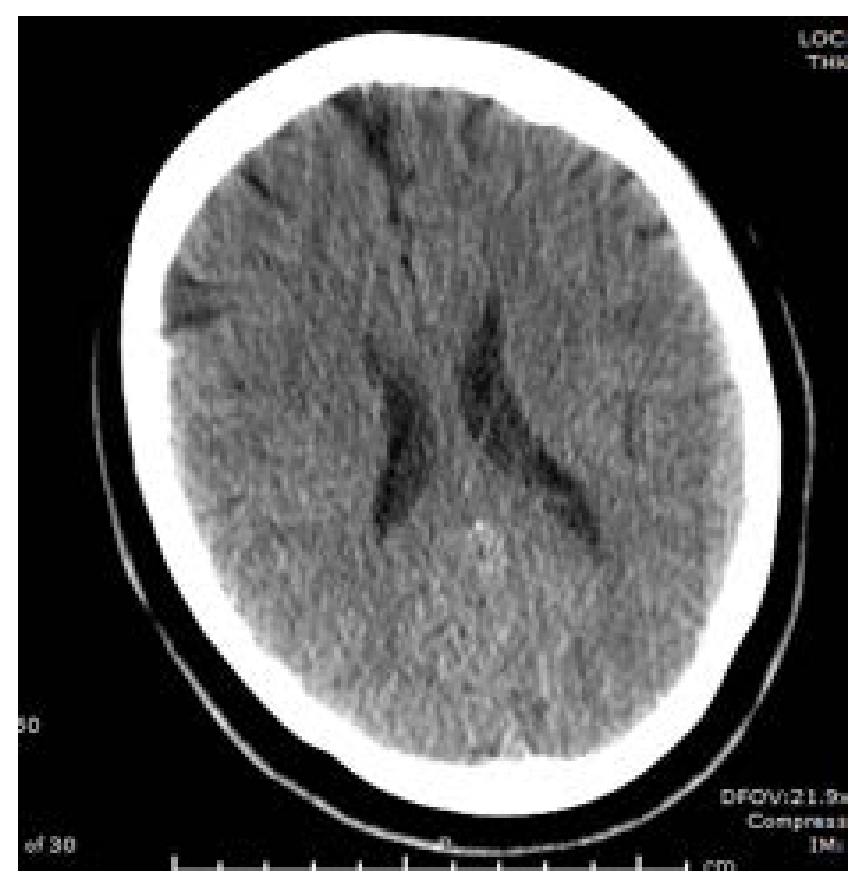

Figure 1. Axial CT demonstrating mild parenchymal volume loss (atrophy) that is greater than expected for the patient's age

Table 1. Laboratory Data

\begin{tabular}{|l|c|c|}
\hline Serum & Patient & Reference Range \\
\hline WBC (K/uL) & $\mathbf{1 7 . 6 5}$ & $4.5-10.9$ \\
\hline RBC (M/uL) & 3.90 & $4.2-5.4$ \\
\hline Hemoglobin (g/dL) & $\mathbf{1 1 . 1}$ & $12.0-16.0$ \\
\hline Hematocrit (\%) & $\mathbf{3 2 . 4}$ & $37.0-47.0$ \\
\hline Platelets (K/uL) & $\mathbf{4 5 2}$ & $130-400$ \\
\hline Sodium (mmol/L) & 138 & $136-146$ \\
\hline Potassium (mmol/L) & 3.8 & $3.5-5.0$ \\
\hline Chloride (mmol/L) & 101 & $98-106$ \\
\hline BUN (mg/dL) & 7 & $6-20$ \\
\hline Creatinine (mg/dL) & 1.01 & $0.4-1.2$ \\
\hline Calcium (mg/dL) & 9.4 & $8.4-10.3$ \\
\hline Total Protein (g/dL) & 8.2 & $6.0-8.5$ \\
\hline Albumin (g/dL) & 3.76 & $2.8-5.7$ \\
\hline AST (U/L) & $\mathbf{1 3 5}$ & $10-35$ \\
\hline ALT (U/L) & $\mathbf{9 7}$ & $0-31$ \\
\hline Alk Phos (U/L) & 109 & $25-125$ \\
\hline Total Bilirubin & 0.90 & $0.0-1.2$ \\
\hline Glucose (mg/dL) & 121 & $70-99$ \\
\hline ESR(mm/h) & $\mathbf{1 0 4}$ & $0-20$ \\
\hline CRP(mg/dL) & $\mathbf{3 2 . 3}$ & $1.0-4.0$ \\
\hline TSH (miU/L) & 1.58 & $0.27-4.20$ \\
\hline Thyroxine (ug/d) & 12.1 & $6-12$ \\
\hline T4 Free (ng/dL) & 1.15 & $1.0-1.53$ \\
\hline Vitamin B12 (pg/mL) & 1070 & $211-946$ \\
\hline Lactate (mmol/L) & $\mathbf{2 . 5}$ & $0.5-2.2$ \\
\hline Cerebrospinal Fluid & & $<5$ \\
\hline WBC /cu mm & 1 & $40-45$ \\
\hline RBC /cu mm & 2 & \\
\hline Lymph /cu mm & 1 & \\
\hline Gram Stain & & \\
\hline Protein (mg/dL) & or & \\
\hline Glucose (mg/dL) & No polys & \\
\hline
\end{tabular}

A lumbar puncture was performed and the results were inconclusive. Chest X-ray did not show any evidence of acute pleural or pulmonary pathology. Urinalysis was positive for leukocyte esterase, nitrites, and $27 \mathrm{WBC} / \mathrm{hpf}$. Urine toxic screen was negative. Other significant labs were elevated liver enzymes, ESR and CRP. Axial head computed tomography demonstrated mild parenchymal volume loss (atrophy) that was greater than expected for the patient's age (Figure 1). The patient's laboratory data is summarized in the table below (Table 1).

The patient's acute mental status change along with signs of sepsis were concerning for possible bacterial or viral meningitis, vancomycin, ceftriaxone and acyclovir were initiated in addition to intravenous fluids for empiric management. A urine culture was obtained after the first dose of antibiotics had been administered. The neurology service was consulted and after a review of cerebrospinal fluid meningoencephalitis panel and brain imaging acyclovir was discontinued. Her condition began to improve however, she continued to exhibit pressured speech, delusions of grandeur and beliefs that she had been sent by God to deliver everyone from the evil forces.

Further history was obtained from relatives. In the previous nine months, the patient had only been sleeping four hours per night, significantly reduce from baseline; she had applied for and was working for multiple jobs due to her increased energy and had gained a substantial amount of weight. On questioning, the patient reported auditory hallucinations ever since she was young, with voices telling her to kill herself. Ten days prior to presentation, the patient had developed urgency and dysuria that worsened over the days accompanied by subjective fevers and odd behavior which included hyposomnia, pressure speech, and agitation. In the hours prior to presentation, the patient had been exhibiting maniac features, delusions of grandeur, and agitation. There was also a history of a depressive episode some years before.

Blood, CSF, and urine cultures all returned negative. A psychiatry consultation was requested and it was determined that the patient's symptoms were consistent with bipolar disorder currently in the manic phase with psychotic features possibly precipitated by her UTI. Neurology consultants recommended an exhaustive work-up to exclude organic causes that could explain the patient's symptoms. The patient's TSH, $\mathrm{T}_{4}, \mathrm{~B}_{12}$, and ACE levels were all within normal limits. Antiphospholipid antibodies and anti-nuclear antibodies returned negative. MRV, MRA, and MRI were obtained. Axial FLAIR image demonstrated a cystic space in the posterior lentiform nucleus consistent with perivascular space (Figure 2). Axial T2 weighted image demonstrated a small hyperintensity in the right ganglio-capsular region (Figure 3). Representing a non-specific area of gliosis possibly due to old ischemia. MRV, MRA and electroencephalogram did not show any significant findings. The psychiatry consultant recommended a trial of olanzapine (10mg at bedtime) however, the patient did not improve and continued to demonstrate grandiosity and deluded ideas that rushed through her mind. Lithium $600 \mathrm{mg}$ was initiated and appropriate blood lithium levels were measured 24 hours later to determine the next appropriate dosing. After 48 hours of having steady 
lithium blood levels, the patient was discharged in improved condition and was prescribed lithium 600mg BID and olanzapine $10 \mathrm{mg}$ daily. Arrangements were made for outpatient follow-up with psychiatry.

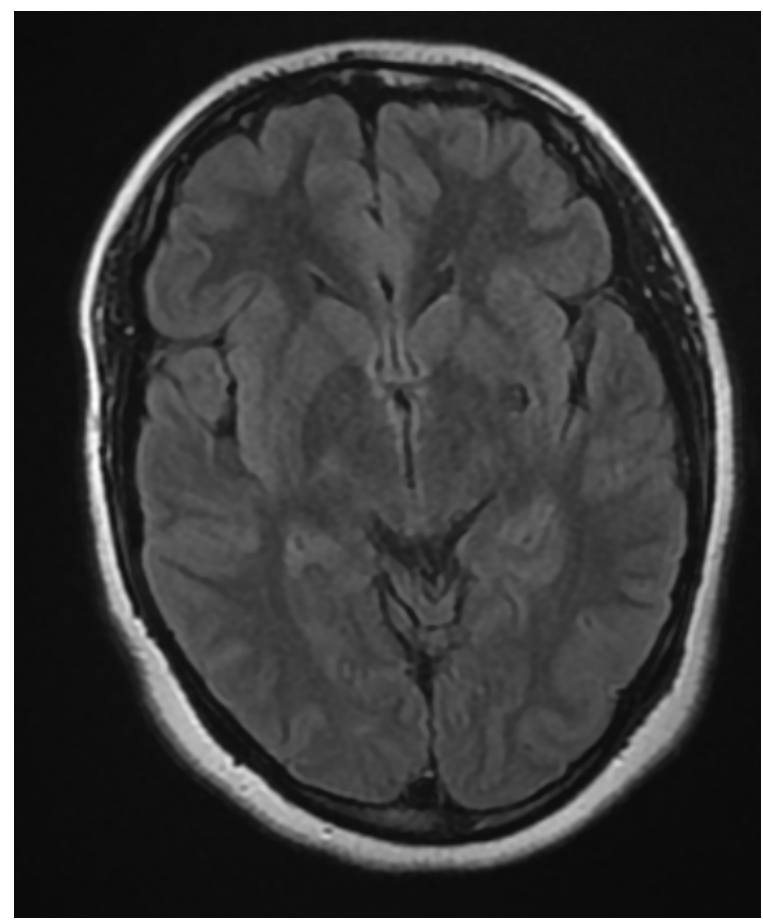

Figure 2. Axial FLAIR image demonstrates a cystic space in the posterior left lentiform nucleus consistent with a perivascular space

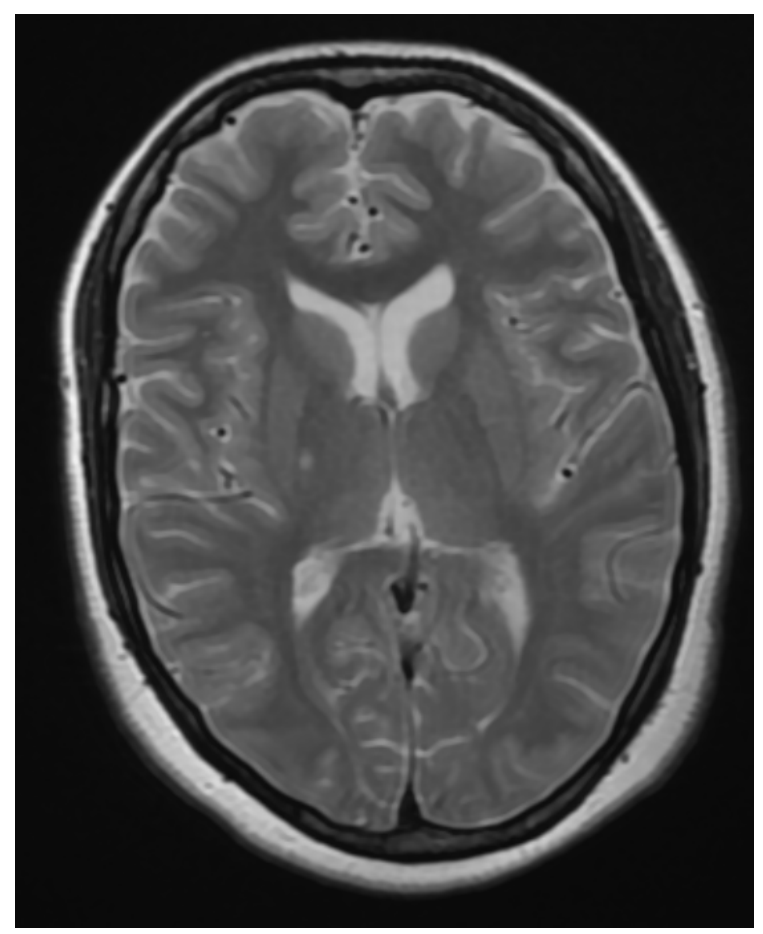

Figure 3. Axial T2 weighted image demonstrates a small Hyperintensity in the right gangliocapsular region

\section{Discussion}

Psychosis is a feature of many neuropsychiatric disorders (most classically schizophrenia). However, psychosis can occur during mood episodes in association with major depressive disorder or bipolar disorder in which case it is referred to as affective psychosis [6].

The natural course of bipolar disorder involves high rates of relapsing and remitting affective episodes. This presents a challenge in terms of treatment as patients may not have a sustained response and may relapse after acute manic episodes [7]. One of the largest unanswered questions in the literature is, what are the underlying pathophysiological factors for these episodes [8]?

Observational studies demonstrated that acute episodes of psychosis could be linked to infections due to bacteria, viruses and protozoa among other pathogens, with improvement of the psychiatric symptoms when the intercurrent infection was resolved [4]. In schizophrenia, the interplay of host's genomics, natural immunity, environmental factors and exposure to pathogens with the ability to alter the normal blood brain barrier by inducing local inflammation is being considered the core pathogenetic mechanism [4].

Urinary tract infections have been described in the setting of various neuropsychiatric conditions including delirium, dementia, psychotic disorders, and mood disorders. A systematic review found that $88 \%$ of the articles reviewed in this area demonstrated a positive association between UTI and neuropsychiatric disorders [9]. In addition, 47\% of these articles described UTI being involved in exacerbating or even precipitating these conditions [9]. There was also an approximate nine times increased prevalence of UTI in patients with mood disorders when compared to controls [10]. Based on this information UTI seem to be a comorbid condition associated with many neuropsychiatric disorders but their role in the pathophysiology of these disorders is unclear. One particularly interesting theory proposed is that the inflammatory response from infection leads to sustained cytokine release that may damage dopaminergic neurotransmission leading to the development of psychosis $[4,10,11]$. Recent research encountered an increased number of macrophages, in the vicinity of neurons, glial cells and endothelial cells, among schizophrenia patients with high levels of inflammation. This novel finding suggest that inflammatory signals could central to the pathogenesis of schizophrenia. Glial cells were also found to have high levels of inflammation which in turn lead to the production of distress signals on endothelial cells, allowing easy migration of the immune cells across the blood brain barrier into the brain tissue [11].

In support of the notion that UTIs are the preceding and precipitating events for the onset of acute psychiatric presentations, the resolution of the urinary infection has also being correlated with the resolution of the psychotic symptoms. There is an implication that infection, UTI being one of the most common, may play a role in the pathophysiology of acute psychotic episodes and the progression of these disease processes $[9,10]$.

A study encountered a higher risk for development of nonaffective psychosis among children from mothers with psychiatric disease who have suffered pre-natal UTI compared to mothers with psychiatric disease who had UTI before the pregnancy. The researchers also found that childhood infections were also more common among these children. The UTI in the pregnancy appeared to affect the offspring at a critical period 
adding to the genetic risk conferred by the maternal disease [3].

Although the current literature refers to psychosis and UTI, the same association has not been firmly established among bipolar disorder patients. However, it is possible that UTIs confer the same risk to bipolar disorder patient population as it has been demonstrated with neuropsychiatric conditions. [9,10]

The patient presented in this case is rather unusual, as she had been a productive well-functioning individual exhibiting the classic signs of mania unbeknownst to her family. It was not until the onset of a UTI that she began to exhibit worsening psychotic symptoms that concerned the family and brought her into the emergency department. Her clinical presentation as well as her hospital course and acute resolution of psychotic symptoms coinciding with resolution of UTI and addition of mood stabilizing therapy, seems to support the notion mentioned above. In addition, her case fills a significant gap in the literature in terms of the relationship between UTI and bipolar disorder with psychotic episodes.

Increased use of imaging, particularly MRI has allowed for greater characterization of structural changes within the brain of patients presenting with psychiatric complaints. Several studies have attempted to uncover the pathophysiology of the changes seen in these patients and their symptomatology. Case reports describing imaging findings also play a key role in helping to further our understanding of the disease process. [12,13,14]

While the patient's findings on imaging are relatively nonspecific there are some interesting things to note. Firstly, the parenchymal volume loss noted on initial CT was greater than expected for the patient's age. This is a finding often seen in psychotic patients, including those affected by schizophrenia, but not usually found in bipolar patients, making this an interesting observation in this particular case. The patient also had prominent perivascular spaces in the posterior lentiform nucleus, commonly known as Virchow-Robin spaces. While these are nonspecific and quite common, especially with the increasing usage of MRI image resolution, it is noteworthy that they have been found to be associated with vascular disease in elderly patients with dementia as well as with diminished cognitive function in patients with cerebral small vessel disease. [15,16]

Finally, the patient was found to have an area of nonspecific gliosis in the medial right basal ganglia. This may represent an old infarction, a curious finding for such a young patient in overall good physical health. A case report compared acute onset of psychosis between those with basal ganglia hemorrhagic stroke and those with midbrain lesions. It has been hypothesized that an infarction in the basal ganglia may lead to impairment of reality-checking pathways in the brain, which leads to psychotic symptoms. While not definitive, it is possible that this gliosis represents an old infarction that could have led to the onset of this patient's bipolar disorder with psychotic features [5,17].

In conclusion, various infections have been recognized to trigger acute episodes of psychosis; UTIs in particular, were found to be more prevalent among patients suffering from acute deterioration of previously stable neuropsychiatric disease. The core mechanism in this pathogenesis of schizophrenia appears to be that infectious pathogens alter endothelial permeability with localized inflammation at the blood brain barrier, thus allowing macrophages and other immune cells and mediators to reach the immune-privileged brain environment. Furthermore, the search for an organic basis for schizophrenia has led to the recognition of a number of imaging patterns: cortical atrophy and gliosis as encountered in our patient have been commonly observed among psychotic patients and could be anatomical evidence for the newly diagnosed psychiatric disorder.

\section{Acknowledgements}

This work is supported in part by Dr. Moro O. Salifu's efforts through NIH Grant \# S21MD012474.

\section{References}

[1] Arias I, Sorlozano A, Villegas E, et al. Infectious agents associated with schizophrenia: a meta-analysis. Schizophr Res. 2012; 136(1-3): 128-136.

[2] Miller BJ, Graham KL, Bodenheimer CM, Culpepper NH, Waller JL, Buckley PF. A prevalence study of urinary tract infections in acute relapse of schizophrenia. J Clin Psychiatry. 2013; 74(3): 271-277.

[3] Blomstrom A, Karlsson H, Gardner R, Jorgensen L, Magnusson C, Dalman C. Associations Between Maternal Infection During Pregnancy, Childhood Infections, and the Risk of Subsequent Psychotic Disorder--A Swedish Cohort Study of Nearly 2 Million Individuals. Schizophr Bull. 2016; 42(1): 125-133.

[4] Severance EG, Yolken RH. From Infection to the Microbiome: An Evolving Role of Microbes in Schizophrenia. Curr Top Behav Neurosci. 2019.

[5] McMurtray A, Tseng B, Diaz N, Chung J, Mehta B, Saito E. Acute Psychosis Associated with Subcortical Stroke: Comparison between Basal Ganglia and Mid-Brain Lesions. Case Rep Neurol Med. 2014; 2014: 428425.

[6] Nugent KL PD, and Ramin Mojtabai R. Non-affective acute psychoses: Uncertainties on the way to DSM-V and ICD-11. Curr Psychiatry Rep. 2011; 13(3): 203-210.

[7] Price AL. Bipolar Disorders: A Review. American Family Physician. 2012; 85(Number 5).

[8] Hilty DM, Leamon MH, Lim RF, Kelly RH, Hales RE. A review of bipolar disorder in adults. Psychiatry (Edgmont). 2006; 3(9): 43-55.

[9] Chae JH, Miller BJ. Beyond Urinary Tract Infections (UTIs) and Delirium: A Systematic Review of UTIs and Neuropsychiatric Disorders. J Psychiatr Pract. 2015; 21(6): 402-411.

[10] Graham KL, Carson CM, Ezeoke A, Buckley PF, Miller BJ. Urinary tract infections in acute psychosis. J Clin Psychiatry. 2014; 75(4): 379-385.

[11] Cai HQ, Catts VS, Webster MJ, et al. Increased macrophages and changed brain endothelial cell gene expression in the frontal cortex of people with schizophrenia displaying inflammation. $\mathrm{Mol}$ Psychiatry. 2018.

[12] Baroud E, Hourani R, Talih F. Brain Imaging in New Onset Psychiatric Presentations. Innov Clin Neurosci. 2019; 16(1-2): 21-26.

[13] Strakowski SM, Delbello MP, Adler CM. The functional neuroanatomy of bipolar disorder: a review of neuroimaging findings. Mol Psychiatry. 2005; 10(1): 105-116.

[14] Pan F WJ, Xu Y, Huan ML. Abnormal parietal encephalomalacia associated with schizophrenia Medicine. 2017; 96(10 (e6310)).

[15] Huijts M, Duits A, Staals J, Kroon AA, de Leeuw PW, van Oostenbrugge RJ. Basal ganglia enlarged perivascular spaces are linked to cognitive function in patients with cerebral small vessel disease. Curr Neurovasc Res. 2014; 11(2): 136-141. 
[16] Patankar TF, Mitra D, Varma A, Snowden J, Neary D, Jackson A. Dilatation of the Virchow-Robin space is a sensitive indicator of cerebral microvascular disease: study in elderly patients with dementia. AJNR Am J Neuroradiol. 2005; 26(6): 1512-1520.

[17] Schnieder TP, Dwork AJ. Searching for neuropathology: gliosis in schizophrenia. Biol Psychiatry. 2011; 69(2):134-139. 